\title{
Frecuencia de la infección por Chlamydia trachomatis en un grupo de mujeres de la Región de la Araucanía, Chile.
}

\author{
Ramón Silva, Daniela León, Tamara Viscarra, Carmen Ili, Juan C. Roa, Raúl Sánchez, Pablo Guzmán y Priscilla Brebi
}

\section{Frequency of Chlamydia trachomatis infection in a group of women from Region of Araucanía, Chile}

Background: Chlamydia trachomatis infection is the most commonly reported sexually transmitted bacterial infection worldwide. Between 70 and $90 \%$ of women are asymptomatic, however, untreated and persistent infections can lead to the development of urethritis, pelvic inflammatory disease, infertility and ectopic pregnancy. Aims: To determine $C$. trachomatis infection frequency in a group of women in Chile, using quantitative real time PCR (qPCR) and to compare the usefulness of endocervical and urine samples for C. trachomatis detection. Methods: 87 asymptomatic women aged 15-64 years were included. Every woman donated one endocervical sample and one urine sample. Detection and quantification of C. trachomatis was performed by qPCR. Results: Of 87 endocervical samples, the frequency was $11.49 \%(\mathrm{n}=10)$. Of these samples, 5 cases were found in women $<35$ years old. About urine samples, 16 samples were positive $(18.39 \%)$. Ten women $<35$ years old yielded positive urine samples. Only four women had both samples positive for C. trachomatis (4.6\%). There was no statistically significant relationship between age and $C$. trachomatis infection. Cryptic plasmid quantification was found between 3.55 - 96.050 copies $/ \mu \mathrm{L}$ for endocervical samples and 7.22-633.1 copies/ $\mu \mathrm{L}$ for urine samples. Conclusion: Estimated frequency of $C$. trachomatis in Chilean women was higher than previous Chilean studies. Both types of samples are complementary for screening and diagnosis strategies using sensitive techniques, because silent infection can be present in either urinary or genital tract or in both in women.

Key words: Chlamydia trachomatis, endocervical sample, urine sample, quantitative real time PCR (qPCR).

Palabras clave: Chlamydia trachomatis, muestra endo-cervical, muestra de orina, RPC en tiempo real cuantitativo (qPCR).

\section{Introducción}

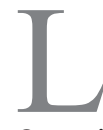

a infección por Chlamydia trachomatis es la infección bacteriana de transmisión sexual más frecuente en el mundo ${ }^{1}$. Según estimaciones de la Organización Mundial de la Salud, cada año se registran 105,7 millones de casos nuevos en todo el mundo, de los cuales 26,4 millones se registran en América ${ }^{1}$. En Chile, la prevalencia registrada en mujeres atendidas en centros ginecológicos, tanto privados como públicos, mediante reacción en cadena de la polimerasa (RPC) y muestras endo-cervicales obtenidas por tórula de algodón, ha sido de $4,7 \%{ }^{2}$.

La infección por C. trachomatis del tracto genital afecta comúnmente a adolescentes y adultos jóvenes, tanto hombres como mujeres ${ }^{3}$. Si bien, esta infección no es causal de muerte de los individuos afectados, su trascendencia radica en la capacidad de estimular la producción persistente de citoquinas pro-inflamatorias, asociadas a un daño permanente en el tejido infectado por la bacteria ${ }^{4-6}$. Por lo tanto, el objetivo del control de $C$. trachomatis está principalmente dirigido a evitar en la mujer consecuencias de la infección persistente ${ }^{7}$, por ejemplo, enfermedad inflamatoria pélvica (EIP) o embarazo ectópico. Estos procesos son secundarios a la inflamación y cicatrización constante de las trompas uterinas (salpingitis) y que incluso puede afectar los ovarios ${ }^{7}$. Sin embargo, la infección por C. trachomatis permanece como una infección silente en 70 a $90 \%$ de las mujeres y en 30 a $40 \%$ de los hombres ${ }^{8}$.

Las muestras más utilizadas para detección molecular de C. trachomatis, han sido las muestras endo-cervicales, que deben ser tomadas por un profesional capacitado, lo que dificulta la realización de tamizaje como estrategia de control. Por otro lado, las muestras de orina, que pueden ser recolectadas por el mismo paciente, y son menos invasoras, son una alternativa para la detección.

Los objetivos de la presente investigación, fueron determinar la frecuencia de la infección por C. trachomatis en un grupo de mujeres de la Región de la Araucanía, Chile, mediante RPC en tiempo real cuantitativa (en inglés la sigla qPCR) y comparar el uso de muestras endo-cervicales y de orina para la detección de $C$. trachomatis.
Universidad de La Frontera, Temuco, Chile. Laboratorio de Virus Oncológicos en Reproducción, BIOREN-CEGIN (RS, DL, TV, RS,).

Laboratorio de Patología Molecular. Departamento de Anatomía Patológica, BIOREN-CEGIN (Cl, PB, PG).

Pontificia Universidad Católica de Chile, Santiago, Chile. Facultad de Medicina Departamento de Patología (JCR)

Recibido: 21 de junio de 2013 Aceptado: 4 de noviembre de 2013

Los autores no poseen conflictos de intereses para la publicación de este artículo.

Esta investigación fue financiada por el Proyecto CORFO N ${ }^{\circ} 12$ IDL218157, Proyecto DIUFRO DI130039, Proyecto CORFO-CEGIN N 09 CN14-5960, Scientific and Technological Bioresource Nucleus (BIOREN); Proyecto FONDECYT Postdoctoral N 3120141 (P. Brebi). Proyecto FONDECYT Postdoctoral No 3130630 (C. Ili).

Correspondencia a: Priscilla Brebi Mieville brebimieville@gmail.com 


\section{Metodología}

\section{Participantes}

Un total de 87 mujeres participaron en este estudio, previa firma de un consentimiento informado. Las mujeres atendidas en el Policlínico de Patología Cervical de Hospital Dr. Hernán Henríquez Aravena de Temuco (n: 62) y en el Consultorio Miraflores de Temuco (n: 25), asistieron a estos establecimientos por control ginecológico de rutina y no informaron síntomas asociados a la infección por C. trachomatis, tales como uretritis, secreción vaginal, cervicitis, u otros. Para establecer una relación entre la frecuencia de infección por C. trachomatis y la edad, se definieron dos grupos etarios: mujeres $<35$ años (n: 44 ) y mujeres $\geq 35$ años (n: 43).

\section{Muestras clínicas}

Se recolectaron dos muestras por cada paciente, una endo-cervical y una de orina. Las muestras endo-cervicales se obtuvieron mediante citobrush y fueron depositadas en $2 \mathrm{~mL}$ de tampón de lisis en tubos de $15 \mathrm{~mL}$. La muestra endo-cervical fue recolectada por profesionales ginecoobstetras. Las muestras de orina fueron recolectadas por cada una de las pacientes, en frascos estériles. Una vez recibidas las muestras, fueron procesadas inmediatamente.

\section{Extracción de ADN desde muestras endo-cervicales}

Cada muestra fue agitada con vórtex para luego transferirla a dos tubos de $1,5 \mathrm{~mL}(1 \mathrm{~mL}$ de muestra a cada tubo). Ambos tubos se centrifugaron a $12.000 \mathrm{rpm}$ por 15 min. a $4^{\circ} \mathrm{C}$ y el pellet se re-suspendió en $500 \mu \mathrm{L}$ de tampón de lisis. Las muestras que mantenían restos celulares fueron digeridas con $2,5 \mu \mathrm{L}$ de proteinasa $\mathrm{K}$ $(20 \mathrm{mg} / \mathrm{mL})$ a $60^{\circ} \mathrm{C}$, durante dos horas. Con posterioridad a la inactivación de proteinasa $\mathrm{K}\left(10\right.$ minutos a $\left.90^{\circ} \mathrm{C}\right)$, se agregó un volumen de $200 \mu \mathrm{L}$ de acetato de amonio $(7,5 \mathrm{M}, \mathrm{pH} 7,5)$ y se centrifugaron a $12.000 \mathrm{rpm}$ por tres minutos a $4^{\circ} \mathrm{C}$. El sobrenadante se traspasó a otro tubo y se le agregó cloroformo $(200 \mu \mathrm{L})$, se mezcló y se incubó a temperatura ambiente por dos min. Se centrifugó a 12.000 rpm por $8 \mathrm{~min}$. a temperatura ambiente. El sobrenadante se transfirió a un nuevo tubo, al cual se le agregó $600 \mu \mathrm{L}$ de isopropanol para precipitar el ADN. Se incubó a $-20^{\circ} \mathrm{C}$ toda la noche. Posteriormente, se centrifugó a $12.000 \mathrm{rpm}$ por $15 \mathrm{~min}$. a $4^{\circ} \mathrm{C}$ y el pellet se lavó con $600 \mu \mathrm{L}$ de etanol $70 \%$, mezclando suavemente. Se centrifugó a $12.000 \mathrm{rpm}$, tres minutos a $4^{\circ} \mathrm{C}$, se eliminó el sobrenadante, y el tubo se dejó destapado hasta secar el pellet. Finalmente, con $30 \mu \mathrm{L}$ de agua ultra pura se rehidrató el pellet. El ADN se almacenó a $-20^{\circ} \mathrm{C}$ hasta su uso.

\section{Extracción de ADN desde muestras de orina}

A un tubo de $15 \mathrm{~mL}$ se adicionó $14 \mathrm{~mL}$ de la muestra de orina y fue centrifugado a $2000 \mathrm{rpm}$ por $15 \mathrm{~min}$. Se eliminó el sobrenadante y al pellet se le agregó $3 \mathrm{~mL}$ de una solución de proteinasa $\mathrm{K}$ y SDS $1 \%$, se mezcló con vórtex y a $56^{\circ} \mathrm{C}$ durante 24 horas se realizó la incubación. Posteriormente, se agregó $5 \mu \mathrm{L}$ de proteinasa $\mathrm{K}$ al $20 \%$, se mezcló con vórtex y se dejó incubando durante 12 horas. Se adicionó $1 \mathrm{~mL}$ de PC-8 y se centrifugó a $10.000 \mathrm{rpm}$ por cinco minutos (se realizó dos veces). El sobrenadante de la fase superior se traspasó a un tubo de $15 \mathrm{~mL}$ y se le agregó $1 \mathrm{~mL}$ de acetato de amonio 7,5 M, $2 \mu \mathrm{L}$ de glicógeno y $8 \mathrm{~mL}$ de etanol al $100 \%$. Se mezcló el tubo por inversión cuatro veces y se incubó a $-20{ }^{\circ} \mathrm{C}$ durante 24 horas. Se centrifugó a $5.000 \mathrm{rpm}$ durante $35 \mathrm{~min}$. a $4^{\circ} \mathrm{C}$ y se eliminó el sobrenadante. Se agregó $10 \mathrm{~mL}$ de etanol $70 \%$, se centrifugó a $4.750 \mathrm{rpm}$ por $15 \mathrm{~min}$. y se eliminó el sobrenadante (estos pasos se hicieron dos veces). Se secaron los tubos destapados e invertidos sobre papel absorbente. Una vez secos los tubos, se resuspendió el pellet en $20 \mu \mathrm{L}$ de tampón Tris-EDTA (LoTE), y se dejó a $4^{\circ} \mathrm{C}$ toda la noche. Finalmente, se transfirió a un nuevo tubo de $1,5 \mathrm{~mL}$. El ADN se almacenó a $-20^{\circ} \mathrm{C}$ hasta su uso.

\section{RPC en tiempo real cuantitativa ( $q P C R)$}

Se utilizó un juego de partidores (partidor forward GGGATTCCTGTAACAACAAGTCAGG y partidor reverse CCTCTTCCCCAGAACAATAAGAACAC) diseñados por McKechnie y cols., 20099. Estos partidores están dirigidos al plásmido críptico de $C$. trachomatis y generan un producto de 208 pares de bases. La mezcla de RPC se realizó para un volumen final de $20 \mu \mathrm{L}$, incluyendo 1 $\mu \mathrm{L}$ de templado. Se preparó utilizando $7,9 \mu \mathrm{L}$ de agua ultra pura, $10 \mu \mathrm{L}$ del mix de SYBR Green y $0,3 \mu \mathrm{L}$ del colorante de referencia ROX 1:500 (Brilliant II SYBR $\AA$ Green QPCR Master Mix with ROX, Stratagene, CA, USA), y los partidores se utilizaron con una concentración final de $0,2 \mu \mathrm{M}$. Para la cuantificación de $C$. trachomatis se realizó una curva estándar con un control de número copias de ADN conocido (AmpliRun ${ }^{\circledR}$ Chlamydia trachomatis DNA control. Vircell, Granada, España). Para la curva se realizaron cinco diluciones con un duplicado cada una ( 12.860 copias $/ \mu \mathrm{L}, 1.286$ copias $/ \mu \mathrm{L}, 128$ copias/ $\mu \mathrm{L}, 12,8$ copias $/ \mu \mathrm{L}$ y 1,28 copias $/ \mu \mathrm{L})$. La programación del termociclador Mx3000P (Stratagene, CA, USA) se indica en la Tabla 1. Como control de calidad de la reacción se utilizaron: como control negativo $\mathrm{ADN}$ genómico humano y como control positivo la dilución 128 copias/ $\mu \mathrm{L}$ de ADN control comercial, además de un control de mix sin ADN (blanco).

\section{Resulltados}

Se determinó la frecuencia de infección asintomática por C. trachomatis en muestras endo-cervicales y de orina de 87 mujeres. 
Se encontraron 10 muestras endo-cervicales positivas para la detección de $C$. trachomatis por qPCR, lo que representa una frecuencia de infección de $11,49 \%$. No se observaron diferencias en la infección por C. trachomatis según grupo etario (Tabla 2).

En las muestras de orina se obtuvieron 16 casos positivos con los que se determinó una frecuencia de infección de $18,39 \%$ para C. trachomatis. En el grupo de mujeres $<35$ años se detectaron 10 muestras de orina positivas para C. trachomatis; en el grupo etario $\geq 35$ años se detectaron 6 muestras positivas (Tabla 2 ).

El número de casos positivos para C. trachomatis, según el centro de atención ginecológica, no tuvo asociación y diferencias estadísticamente significativas (Tabla 3 ).

Respecto a la correlación de las muestras, sólo en cuatro mujeres, tanto la muestra endo-cervical como la muestra de orina, fueron positivas para la detección de $C$. trachomatis, (4,6\% del total de pacientes).

La cuantificación del plásmido críptico de C. trachomatis, para muestras endo-cervicales, resultó en un rango de 3,55 - 96.050 copias/ $\mu \mathrm{L}$. En las muestras de orina, la cuantificación se encontró en un rango de 7,22 - 633,1 copias $/ \mu \mathrm{L}$.

\section{Discusión}

En este estudio se determinó una frecuencia de 11,49\% de C. trachomatis en un grupo de 87 mujeres utilizando muestras endo-cervicales. Este porcentaje es mayor que el reportado en un anterior estudio chileno efectuado por A. Ovalle y cols., el cual mostró una frecuencia de $5,9 \%$ (n: 255), utilizando muestras cervico-vaginales y realizando la detección mediante RPC anidada. El grupo estudiado por Ovalle incluyó mujeres embarazadas con y sin síntomas de infección, en un rango 14 a 43 años de edad (27,7 años promedio $)^{10}$. Otro estudio chileno, de M. Martínez y cols., incluyó, solamente, muestras endo-cervicales de 403 mujeres, de las cuales 100 se atendieron en el Servicio Ginecológico del Hospital San José (promedio de edad de 35,6 años), 268 mujeres en el Centro Ginecológico privado del Área Metropolitana (promedio de edad de 33,4 años) y 35 mujeres en el Centro Reproductivo de Adolescentes (promedio de años 16,8 años). La detección de C. trachomatis se realizó por RPC convencional y para la confirmación de casos positivos se utilizó RPC anidada. En total, la frecuencia obtenida fue de 4,7\% (n: 19). No hubo diferencias significativas entre los grupos etarios y la prevalencia. Sin embargo, la prevalencia determinada en el Centro Reproductivo de Adolescentes (8,6\%) y el Servicio Ginecológico del Hospital San José ( $2 \%$ ), en el cual las pacientes son adultas, difirió de forma significativa $(\mathrm{p}=0,0382)^{2}$. En relación a estudios latinoamericanos, en una población de mujeres
Tabla 1. Programa de RPC en tiempo real cuantitativa (qPCR)

\begin{tabular}{|lccc|}
\hline Etapa & Temperatura & Tiempo & $\mathbf{n}$ de ciclos \\
Activación & $95^{\circ} \mathrm{C}$ & 10 minutos & 1 \\
Denaturación & $95^{\circ} \mathrm{C}$ & 30 segundos & \\
Alineamiento & $60^{\circ} \mathrm{C}$ & 30 segundos & 40 \\
Extensión & $72^{\circ} \mathrm{C}$ & 30 segundos & \\
Curva de disociación & $95^{\circ} \mathrm{C}$ & 1 minuto & \\
& $55^{\circ} \mathrm{C}$ & 30 segundos & 1 \\
& $95^{\circ} \mathrm{C}$ & 30 segundos & \\
\hline
\end{tabular}

Tabla 2. Casos positivos para Chlamydia trachomatis, según grupo etario y tipo de muestra

\begin{tabular}{llcc} 
Grupo etario & $\begin{array}{c}\mathbf{n} \text { mujeres } \\
(\%)\end{array}$ & $\begin{array}{c}\mathbf{n} \text { muestras endocervicales } \\
\text { positivas }(\%)\end{array}$ & $\begin{array}{c}\mathbf{n} \text { muestras de orina } \\
\text { positivas }(\%)\end{array}$ \\
$<35$ años & $44(50,57)$ & $5(11,36)$ & $10(22,72)$ \\
$\geq 35$ años & $43(49,43)$ & $5(11,62)$ & $6(13,95)$ \\
Total & $87(100)$ & $10(11,49)$ & $16(18,39)$ \\
\hline
\end{tabular}

Tabla 3. Casos positivos para Chlamydia trachomatis, según centro de atención ginecológica y tipo de muestra

\begin{tabular}{|lccc}
$\begin{array}{l}\text { Centro de atención } \\
\text { ginecológica }\end{array}$ & $\begin{array}{c}\mathbf{n} \text { mujeres } \\
(\%)\end{array}$ & $\begin{array}{c}\mathbf{n} \text { muestras endocervicales } \\
\text { positivas (\%) }\end{array}$ & $\begin{array}{c}\mathbf{n} \text { muestras de orina } \\
\text { positivas (\%) }\end{array}$ \\
$\begin{array}{l}\text { Consultorio Miraflores } \\
25(28,74)\end{array}$ & $5(20)$ & $9(36)$ \\
$\begin{array}{l}\text { Policlínico de patología } \\
\text { cervical }\end{array}$ & $62(71,26)$ & $5(8,06)$ & $7(11,3)$ \\
Total & $87(100)$ & $10(11,49)$ & $16(18,39)$ \\
\hline
\end{tabular}

brasileras de un rango etario de 14 a 63 años (n: 121), utilizando muestras endo-cervicales, se demostró $20,79 \%$ de casos positivos mediante RPC convencional, destacando que $80 \%$ de los casos positivos perteneció a mujeres de 23 años o menos ${ }^{11}$. Otro estudio en Brasil, también con RPC convencional como sistema de detección, en una población femenina sexualmente activa de 10 a 19 años (n: 100), se determinó una prevalencia de infección de $C$. trachomatis en $31 \%$ de muestras endo-cervicales ${ }^{12}$. Por otro lado, en muestras endo-cervicales de 105 mujeres venezolanas (18 a 48 años), tanto sintomáticas como no sintomáticas, determinó una prevalencia de 10,4\% de infección por C. trachomatis. Específicamente, en las muestras provenientes de mujeres asintomáticas (n: 54), la frecuencia de casos positivos fue de $7,4 \%{ }^{13}$.

En el presente estudio, además, se determinó la frecuencia de infección por $C$. trachomatis en muestras de orina de las mismas pacientes que donaron la muestra endo-cervical. Debido a que el uso de orina para detección 
de C. trachomatis no es común, pocos estudios latinoamericanos han reportado prevalencia de la infección utilizando este tipo de muestra. En nuestro estudio, la prevalencia fue de $18,39 \%$, mayor a la reportada en Chile previamente, donde se detectó $6,9 \%$ casos por RPC convencional en muestras de 203 mujeres de 12 a 25 años de edad, sexualmente activas. Además, en esta población de mujeres, 104 de ellas pertenecían al grupo socio-económico medio-alto ( $3,8 \%$ casos positivos) y 99 al grupo socio-económico bajo (10,1\% casos positivos), entre los cuales no hubo diferencia significativa $($ Fisher $=0,068)$. Por otro lado, en mujeres embarazadas, se detectó 19\% de casos positivos y en las no embarazadas sólo 5,5\%, lo cual fue estadísticamente significativo $(\text { Fisher }=0,043)^{14}$. Estos resultados en muestras de orina son similares a los encontrados en México, con una prevalencia de $4,81 \%$ en un grupo de jóvenes (hombres y mujeres) utilizando la técnica de reacción en cadena de la ligasa (sigla en inglés: LCR $)^{15}$. En Colombia, en un estudio realizado mediante RPC en orina se observó 4 y 4,5\% de C. trachomatis en mujeres con parto prematuro y con ruptura prematura de membrana, respectivamente ${ }^{16}$.

Es importante señalar que en Chile, no se han publicado con anterioridad análisis de prevalencia de $C$. trachomatis utilizando la técnica de RPC en tiempo real. Por esta razón, se justifican los porcentajes de casos positivos obtenidos de $11,46 \%$ y $18,39 \%$, en algunos casos mayores a estudios similares en Latinoamérica, ya que la detección se realiza con un método de alta sensibilidad, con resultados altamente confiables y menor riesgo de contaminación.

En relación a la sensibilidad y especificidad asociado al tipo de muestra y el sistema de detección, no deberían existir diferencias entre las muestras de orina y endo-cervicales, según lo revisado por Cook y $\mathrm{cols}^{17}$. En el presente estudio, la frecuencia de infección por $C$. trachomatis fue mayor en muestras de orina (18,39\%), lo que podría deberse a que el proceso inflamatorio es más común a nivel uretral en este grupo de estudio. Aunque no es posible descartar la contaminación de la orina con secreción vaginal, ya que la muestra es recolectada por el propio paciente, en este estudio se recolectaron de una misma paciente una muestra endo-cervical y una muestra de orina, por lo que se considera que los casos positivos en orina provienen exclusivamente de la vía uretral, a excepción de cuatro casos que fueron positivos para ambas muestras y se discuten más adelante. De manera que, como es sabido, C. trachomatis puede causar infecciones del aparato genital femenino, como vaginitis o cervicitis, y además puede infectar las vías urinarias, ocasionando uretritis no gonocóccica ${ }^{18}$. Por lo tanto, el uso de la muestra de orina es de gran importancia para detección de esta bacteria, e incluso podría ser usada para la búsqueda de esta infección en hombres.
Cabe señalar que, la correlación entre la muestra de orina y la muestra endo-cervical obtenida por citobrush para la detección C. trachomatis fue sólo en cuatro mujeres que presentaron ambas muestras positivas $(4,6 \%)$. Considerando que el resto de los casos positivos estaban presentes en un solo tipo de muestra, para fines diagnósticos de $C$. trachomatis, ambas muestras son complementarias, especialmente, para pacientes asintomáticas, ya que $C$. trachomatis puede infectar la vía urinaria, tracto genital o ambos.

Si bien, la sensibilidad de la técnica de RPC depende de la calidad de la muestra, del sistema de detección del producto o de la eficiencia propia de RPC, en este trabajo fue posible aumentar la sensibilidad utilizando partidores dirigidos al plásmido críptico bacteriano, debido a que una bacteria puede contener alrededor de 10 copias de plásmido críptico ${ }^{19}$. Así, se puede cuantificar un mínimo de 3,55 copias $/ \mu \mathrm{L}$, lo que significaría la presencia de una bacteria. En otros estudios, la cuantificación de $C$. trachomatis utilizando un gen presente en el cromosoma bacteriano en una sola copia $(\mathrm{ompl})$, registró una carga bacteriana promedio de 5,6 x $10^{6}$ copias/muestra en un rango de 4,2 × $10^{3}-2,6 \times 10^{9}$ copias/muestra en especímenes vaginales ${ }^{20}$. En la cuantificación de $C$. trachomatis utilizando el gen omp $A$, tanto en muestras urogenitales féminas como masculinas, se encontró una mayor carga bacteriana en mujeres, con un promedio de 5,6 $\log \left(4 \times 10^{5}\right.$ copias/muestra) versus 3,5 $\log \left(3,2 \times 10^{4}\right.$ copias/muestra) en muestras masculinas ${ }^{21}$.

Finalmente, es importante resaltar la necesidad de implementar estrategias eficientes de tamizaje y tratamiento de la infección por C. trachomatis. El presente estudio, realizado en mujeres asintomáticas, reafirma el estado silente de la infección por C. trachomatis, determinando una frecuencia de infección de $11,49 \%$ a nivel cervical. El $50 \%$ de estos casos positivos correspondieron a mujeres $<35$ años. Esto podría implicar que, si esta infección no es tratada, puede progresar a daños en los tejidos del aparato reproductor, producto del proceso inflamatorio que acompaña a esta infección, y que está asociado a embarazo ectópico e infertilidad en mujeres en edad reproductiva.

\section{Resumen}

Introducción: La infección por Chlamydia trachomatis es la infección bacteriana de transmisión sexual más frecuente en el mundo. Entre 70 y $90 \%$ de las mujeres son asintomáticas; sin embargo, las infecciones sin tratar y persistentes permiten el desarrollo de uretritis, enfermedad inflamatoria pélvica, infertilidad y embarazo ectópico. Objetivos: Determinar la frecuencia de infección por $C$. trachomatis en un grupo de mujeres chilenas, mediante $\mathrm{RPC}$ en tiempo real cuantitativa (qPCR) y comparar la 
utilidad de muestras endo-cervicales y de orina para la detección de C. trachomatis. Metodología: Participaron 87 mujeres asintomáticas (15-64 años). Cada mujer donó una muestra endo-cervical y una de orina. Se realizó detección y cuantificación C. trachomatis mediante qPCR. Resultados: La frecuencia de infección por C. trachomatis en muestras endo-cervicales fue de $11,49 \%$ (n: 10 ) y en muestras de orina de 18,39\% (n: 16). El mayor número de casos se encontró en mujeres $<35$ años. Sólo en cuatro mujeres se detectó $C$. trachomatis en ambas muestras $(4,6 \%)$. La cuantificación de plásmido críptico se encontró en un rango 3,55 - 96.050 copias/ $\mu \mathrm{L}$. Conclusión: La frecuencia estimada de $C$. trachomatis fue más alta que en otros estudios chilenos. Ambos tipos de muestra deberían ser complementarias para estrategias de tamizaje y diagnóstico de $C$. trachomatis usando técnicas sensibles de detección, ya que la infección puede desarrollarse en el tracto genital y/o en el tracto urinario en mujeres.

\section{Referencias bibliográficas}

1.- Global incidence and prevalence of selected curable sexually transmitted infections-2008. WHO 2012. http://apps.who.int/iris/ bitstream/10665/75181/1/9789241503839_eng. pdf

2.- Martínez M, Reid I, Arias C, Napolitano C, Sandoval J, Molina R. Prevalencia de infección cervical por Chlamydia trachomatis en mujeres de la Región Metropolitana. Rev Med Chile 2008; 136: 1294-300.

3.- CDC. CDC grand rounds: Chlamydia prevention: 202 challenges and strategies for reducing disease burden and sequelae. MMWR Morb Mortal Wkly Rep 2011; 60 (12): 370-3. http://www.cdc.gov/mmwr/pdf/wk/mm6012.pdf

4.- Rasmussen SJ, Eckmann L, Quayle A J, Shen L, Zhang Y X, Anderson D J, et al. Secretion of proinflammatory cytokines by epithelial cells in response to Chlamydia infection suggests a central role for epithelial cells in chlamydial pathogenesis. J Clin Invest 1997; 99(1): 77-87.

5.- Maxion H, Kelly K. Chemokine expression patterns differ within anatomically distinct regions of the genital tract during Chlamydia trachomatis infection. Infect Immun 2002; 70(3): 1538-46.

6.- Johnson R. Murine oviduct epithelial cell cytokine responses to Chlamydia muridarum infection include interleukin-12-p70 secretion. Infect Immun 2004; (7): 3951-60.

7.- Paavonen J, Eggert-Kruse W. Chlamydia trachomatis: impact on human reproduction. Hum Reprod Updat 1999; 5: 433-47.

8.- Peipert J. Clinical practice. Genital chlamydial infections. N Engl J Med 2003; 349: 2424-30.

9.- McKechnie M L, Hillman R, Couldwell D, Kong F, Freedman E, Wang H, et al. Simultaneous identification of 14 genital microorganisms in urine by use of a multiplex PCR-based reverse line blot assay. J Clin Microbiol 2009; 47 (6): 1871-7.

10.- Ovalle A, Martínez M A, Fuente F De, Falcon N, Feliú F, Fuentealba F. Prevalencia de infecciones de transmisión sexual en mujeres embarazadas atendidas en un hospital público de Chile. Rev Chilena Infectol 2011; 28: 517-20.

11.- Santos C, Teixeira F, Vicente A, Astolfi-Filho S. Detection of Chlamydia trachomatis in endocervical smears of sexually active women in Manaus-AM, Brazil, by PCR. Braz J Infect Dis 2003; 7 (2): 91-5.

12.- Machado M S C, Costa e Silva B F B Da, Gomes I L C, Santana I U, Grassi M F R. Prevalence of cervical Chlamydia trachomatis infection in sexually active adolescents from Salvador, Brazil. Braz J Infect Dis 2012; 16 (2): 188-91.

13.- Arráiz N, Ginestre M, Perozo A, Castellano M, Urdaneta B, García M. Diagnóstico molecular y prevalencia de infecciones por Chlamydia trachomatis en pacientes sintomáticas y asintomáticas de una población del estado de Zulia, Venezuela. Rev Chilena Infectol 2007; 24 (1): 48-52.

14.- Huneeus A, Pumarino MG, Schilling A, Robledo P, Bofil M. Prevalencia de Chlamydia trachomatis y Neisseria gonorrhoeae en adolescentes chilenas. Rev Med Chile 2009; 137: 1569-74.

15.- Sánchez-Alemán M Á, Gutiérrez J P, Bertozzi S M, Frontela-Noda M, Guerrero-
Lemus V, Conde-González CJ. Detección de Chlamydia trachomatis en orina por LCR: aplicación del método de mezcla de muestras biológicas. Rev Invest Clin 2005; 57 (4): 548-54.

16.- Ostos O, Angel E, Bonilla H, Cifuentes $\mathrm{C}$, Correa C. Estudio piloto de prevalencia de infección por Chlamydia trachomatis detectada por PCR en mujeres con parto prematuro en el Instituto Materno Infantil de Bogotá. Rev Colomb Obstet Ginecol 2005; 56 (3): 225-30.

17.- Cook R L, Hutchison S L, Ostergaard L, Braithwaite R S, Ness R B. Systematic review: noninvasive testing for Chlamydia trachomatis and Neisseria gonorrhoeae. Ann Intern Med. 2005; 142 (11): 914-25.

18.- Darville T, Hiltke T. Pathogenesis of genital tract disease due to Chlamydia trachomatis. J Infect Dis 2010; 201: S114-S125.

19.- Fredlund H, Falk L, Jurstrand M, Unemo M. Molecular genetic methods for diagnosis and characterisation of Chlamydia trachomatis and Neisseria gonorrhoeae: impact on epidemiological surveillance and interventions. APMIS. 2004; 112 (11-12): 771-84.

20.- Walker J, Fairley C K, Bradshaw C S, Tabrizi S N, Chen M Y, Twin J, et al. "The difference in determinants of Chlamydia trachomatis and Mycoplasma genitalium in a sample of young Australian women". BMC Infect Dis. BioMed Central Ltd 2011; 11 (1): 35.

21.- Jalal H, Verlander N Q, Kumar N, Bentley N, Carne C, Sonnex C. Genital chlamydial infection: association between clinical features, organism genotype and load. J Med Microbiol 2011. 